\title{
Unusual Presentation of Brucellosis in Baker's Cyst
}

\author{
Osama Al Shaya ${ }^{\mathrm{a}}$, Muhammed Raza Amin ${ }^{\mathrm{a}}$, Mohammed Benmeakel ${ }^{\mathrm{b}, \mathrm{c}}$
}

\begin{abstract}
A 55-year-old diabetic male came with history of left knee pain and swelling for 7 days, fever for 3 days, and no history of trauma. He was complaining of tenderness on the left popliteal fossa, and the surrounding area was red and soft to firm swelling; he was limping, and could not bear weight on the left leg. The ultrasound showed that there was a well-defined heterogeneous hypoechoic cystic lesion. Under aseptic technique, continuous US guidance and local anesthesia, we aspirated almost all the fluid of the left calf collection, which was reported as complicated baker cyst. The patient was put on ciprofloxacin, gentamycin, and doxycycline. The patient was cleared from infectious diseases' team and discharged after 7 days.
\end{abstract}

Keywords: Baker's cyst; Brucellosis; Knee

\section{Introduction}

Brucellosis is a zoonotic infection, transmitted to humans by contact with infected animals and their products such as unpasteurized milk, animals such as sheep, cattle, and goats. Brucellosis has very broad clinical spectrum ranging from asymptomatic to very severe systemic manifestations [1].

The prevalence of brucellosis has been increasing in the developing countries especially the golf countries (KSA, Qatar, etc.) [2]. In one study, the incidence rate was 34/100,000 in Tabuk Province of KSA [3].

The brucellosis can present as a focal infection occurring in about $30 \%$ of cases [4]. One of the focal infections is osteoarticular involvement through a hematogenous spread; the sacroiliac joints and knee joint are most frequently involved.

Manuscript accepted for publication September 07, 2015

aKing Fahad Medical City, Riyadh, Saudi Arabia

bKing Saud bin Abdulaziz University for Health Sciences, Riyadh, Saudi Arabia

${ }^{\mathrm{c} C o r r e s p o n d i n g ~ A u t h o r: ~ M o h a m m e d ~ B e n m e a k e l, ~ K i n g ~ S a u d ~ b i n ~ A b d u l a z i z ~}$

University for Health Sciences, Riyadh, Saudi Arabia.

Email:m.meakel@gmail.com

doi: http://dx.doi.org/10.14740/jmc2252w
We report a unique case of a Brucella species that was isolated from a complicated baker's cyst.

\section{Case Report}

A 55-year-old diabetic male came with history of left knee pain and swelling for 7 days, and fever for 3 days. He did not report any history of trauma.

On examination, blood pressure is $150 / 90 \mathrm{~mm} \mathrm{Hg}$, heart rate is $108 / \mathrm{min}$, and respiratory rate is $22 / \mathrm{min}$.

The patient had tenderness on the left popliteal fossa, and the surrounding area was red and soft to firm swelling; he was limping, and could not bear weight on the left leg. It was difficult to palpate popliteal pulse, but distal pulses were palpable. There were no obvious signs of deep venous thrombosis; the rest of the exam was unremarkable.

\section{Investigation}

On lab workup (Table 1), the results were all in normal range except for ESR, which was 52 (RR $0-20 \mathrm{~mm} / \mathrm{h}$ ). X-rays (Fig. 1) showed normal osteoarticular appearance of the knee except for the moderate osteoarthritis. The US (Fig. 2) showed that there was a well-defined heterogeneous hypoechoic cystic lesion measuring about $1.20 \times 1.8 \mathrm{~cm}$ in left popliteal fossa, which appears to be communicating with well-defined heterogeneous hypoechoic with lace-like appearance fluid collection in the medial aspect of the gastrocnemius muscle superficially measuring about $6.6 \times 2 \mathrm{~cm}$ with associated increase in vascularity noted. Finding most likely represented a Baker's cyst

Table 1. Lab Results

\begin{tabular}{lll}
\hline Test code & Result & Reference value \\
\hline WBC & $10.95 \times 10^{9} / \mathrm{L}$ & $3.90-11.00 \times 10^{9} / \mathrm{L}$ \\
Neutrophils, \% & $65.70 \%$ & $30-70 \%$ \\
Lymphocytes, \% & $25 \%$ & $23-60 \%$ \\
Eosinophiles, $\%$ & $0.50 \%$ & $1-12 \%$ \\
ESR & $52 \mathrm{~mm} / \mathrm{h}$ & $0-20 \mathrm{~mm} / \mathrm{h}$ \\
\hline
\end{tabular}

All in normal range except for ESR, which was 52 (RR 0 - 20 mm/h). 

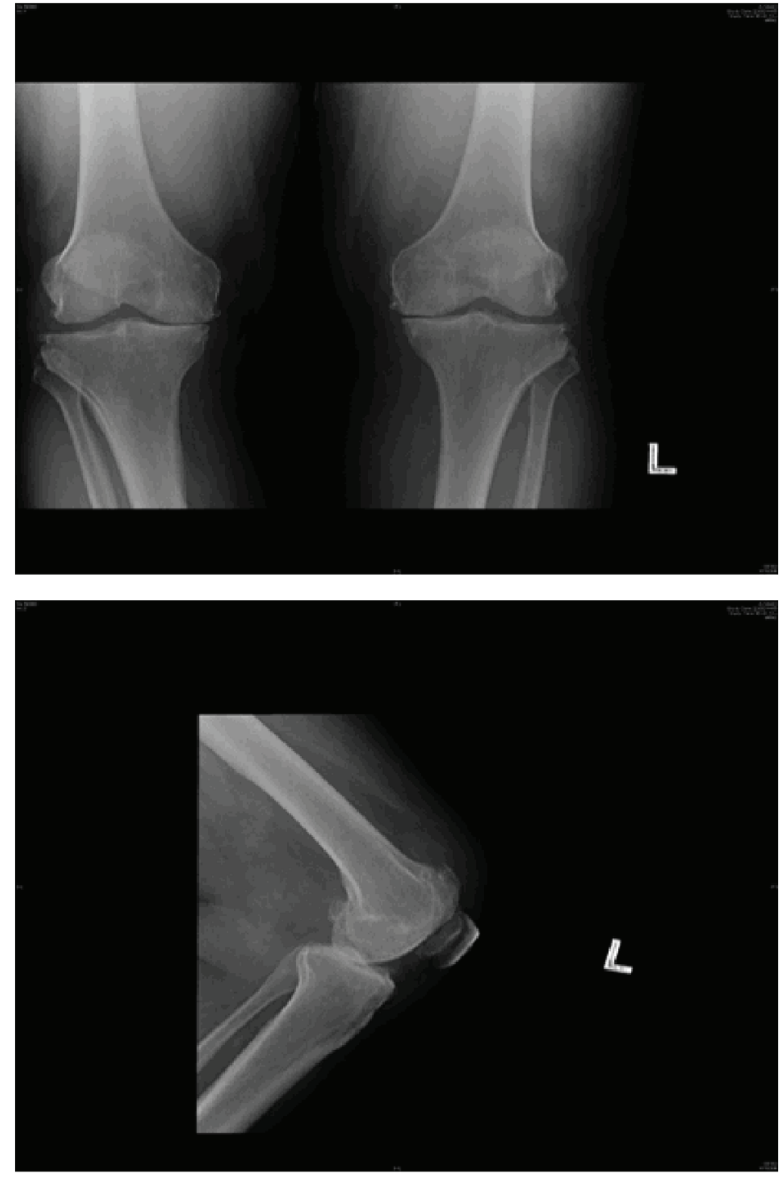

Figure 1. Normal osteoarticular appearance of both knees, except for the moderate osteoarthritis. rupture with the intracystic hemorrhage for clinical correlation.

Under aseptic technique, continuous US guidance and local anesthesia, we aspirated almost all the fluid of the left calf collection, which was reported as complicated baker cyst (Fig. 2).

The fluid showed few WBCs were detected and the Gram smear of enrichment broth showed isolated presence of Brucella species.

\section{Treatment and follow-up}

In hospital course, the patient was admitted and we consulted infectious disease team. The patient was put on ciprofloxacin, gentamycin, and doxycycline, and discharged after 7 days.

On follow-up, he was afebrile; on examination, there were no palpable swelling and full weight bearing. On ultrasound (Fig. 3), there is interval decrease in the size of the previously noted ruptured baker's cyst with intracystic hemorrhage, currently measuring $2.5 \times 0.8 \mathrm{~cm}$, extending from left popliteal fossa to left calf. On MRI (Fig. 4), left knee effusion with synovitis could be inflammatory or infective with large joint effusion without bone destruction. Lab showed ESR is 6 (RR 0 - $20 \mathrm{~mm} / \mathrm{h}$ ).

\section{Discussion}

One of the most frequent presentations of brucellosis is arthritis. In one series study of osteoarticular brucellosis, the knee was the most common site $(61.8 \%$ of the cases) [1].

However, there were no reported cases about brucellosis being a cause of Baker's cyst, except for brief case reported in 1996, where a 60-year-old male, previously healthy with his-

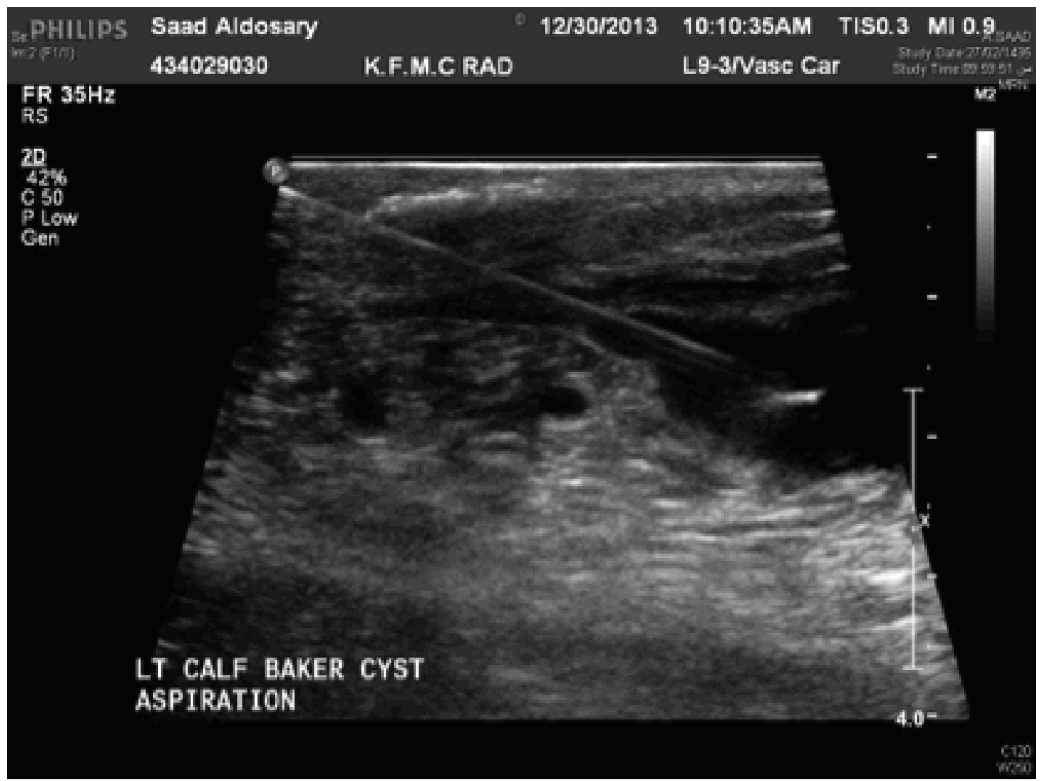

Figure 2. There is a well-defined heterogeneous hypoechoic cystic lesion, and here it is showing the needle is inserted for aspiration. 


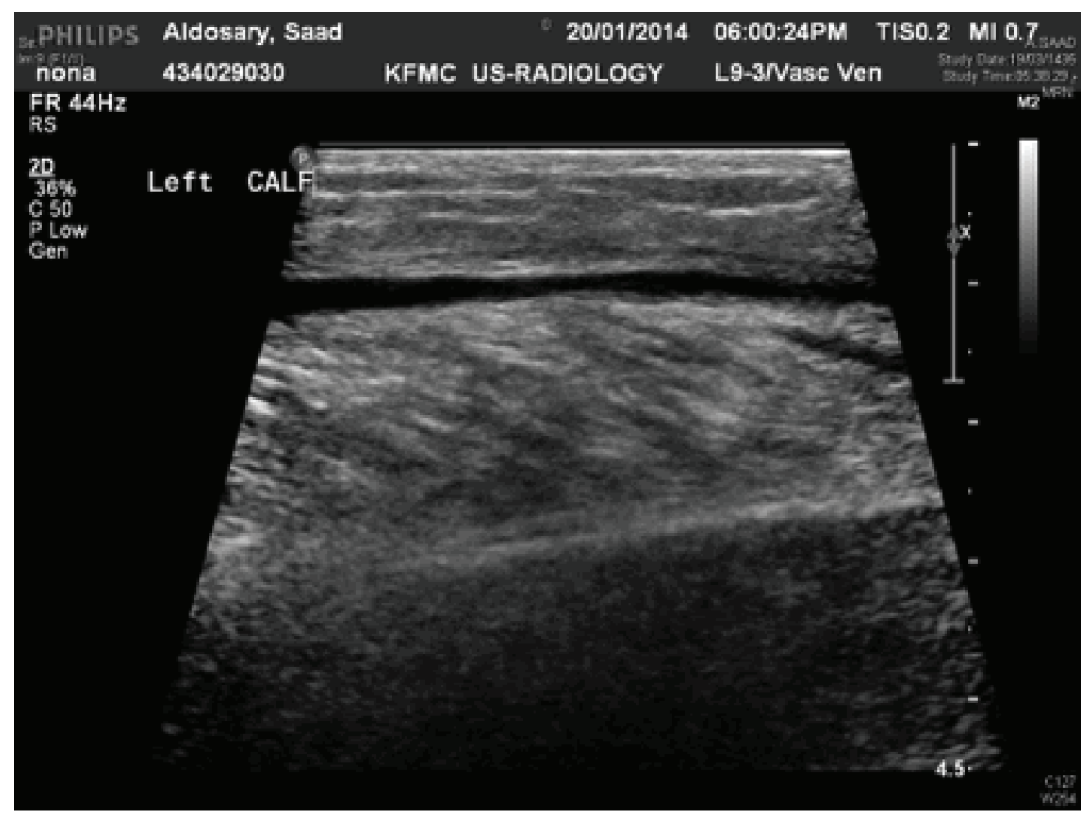

Figure 3. This US has been taken 10 days after aspiration.
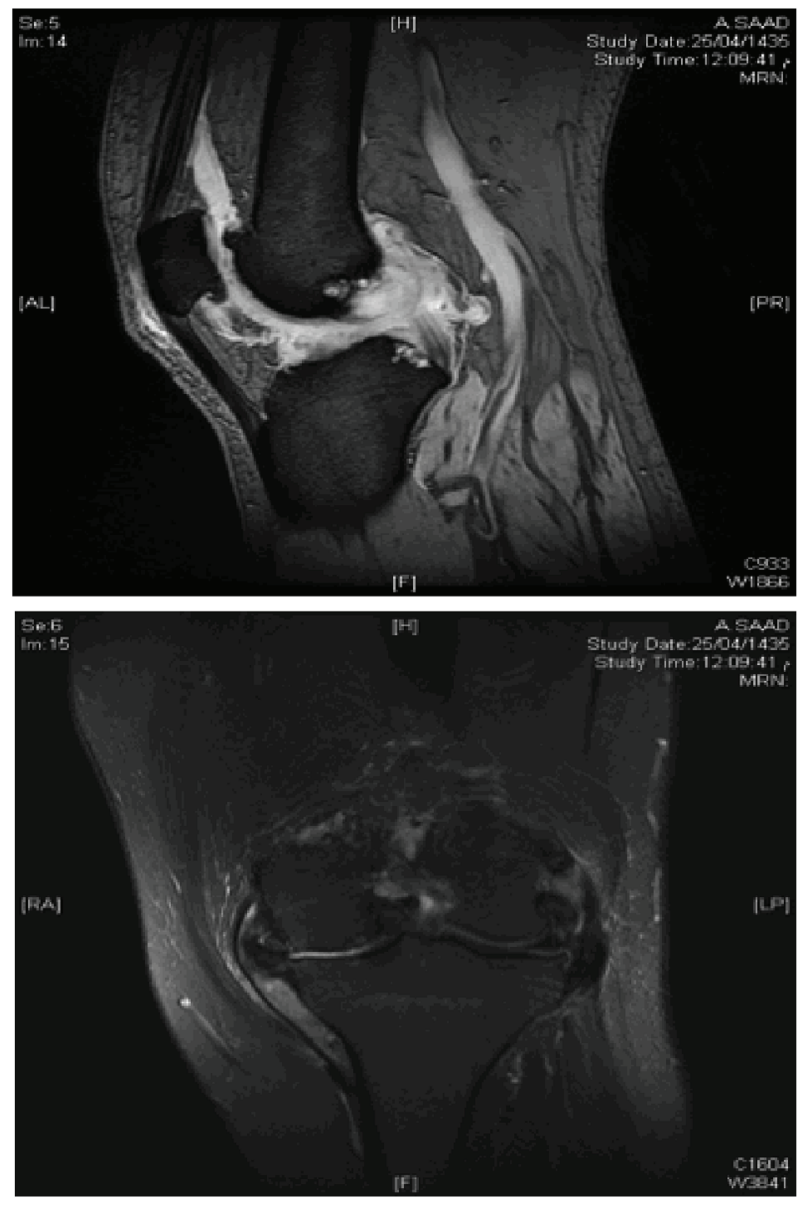

Figure 4. Left knee effusion with synovitis with large joint effusion without bone destruction. tory of drinking raw milk, presented with night sweats, fever and popliteal swelling for 5 months [5]. Comparing this patient to ours, the ESR is $85 \mathrm{~mm} / \mathrm{h}$ while our patient is $52 \mathrm{~mm} / \mathrm{h}$.

Baker's cyst can be a first sign of Aspergillus fumigatus infection but not brucellosis [6]. It was unique when we detected the organism from the drainage with no sign of any hematogenous spread, neither clinically nor on laboratory investigations.

\section{References}

1. Madkour M. Madkour's Brucellosis. Springer; 2001. Available at: http://books.google.com.sa/ books?id=KaNrAAAAMAAJ.

2. Mantur BG, Amarnath SK, Shinde RS. Review of clinical and laboratory features of human brucellosis. Indian J Med Microbiol. 2007;25(3):188-202.

3. Elbeltagy KE. An epidemiological profile of brucellosis in Tabuk Province, Saudi Arabia. East Mediterr Health J. 2001;7(4-5):791-798.

4. Colmenero JD, Reguera JM, Martos F, Sanchez-DeMora D, Delgado M, Causse M, Martin-Farfan A, et al. Complications associated with Brucella melitensis infection: a study of 530 cases. Medicine (Baltimore). 1996;75(4):195-211.

5. Blanco R, Gonzalez-Gay MA, Varela J, Monte R, Sanchez-Andrade A, Gonzalez-Vela C. Baker's cyst as a clinical presentation of brucellosis. Clin Infect Dis. 1996;22(5):872-873.

6. Austin KS, Testa NN, Luntz RK, Greene JB, Smiles S. Aspergillus infection of total knee arthroplasty presenting as a popliteal cyst. Case report and review of the literature. J Arthroplasty. 1992;7(3):311-314. 